J. Acoust. Soc. Jpn. (E) 19, 2 (1998)

\section{Validity of the vibro-acoustic reciprocity method for the measurement of the mechano-acoustical transfer function}

Keywords : Underwater acoustics, Vibro-acoustic reciprocity, Noise transmission, Sound transfer function

PACS number: 43. 20. Tb, 43. 30. Jx, 43. 30. Xm, 43. 40. At, 43. 40. Rj

Takaaki Musha and Tatsuo Kikuchi 5th Research Center, TRDI, Japan Defense Agency, 3-13-1, Nagase, Yokosuka, 239-0826 Japan

(Received 3 September 1997)

\section{Introduction}

For evaluating radiation efficiency of sound from ship structures into surrounding water, it is necessary to know the mechano-acoustical transfer function $H$, which is describes as follows ${ }^{1)}$ :

$$
H=p / F,
$$

where $F$ is the force exerted on the ship structure and $p$ is the underwater sound pressure radiated from it. But it is hard to measure this factor by direct excitation on the ship structures like installed machinery and the ship's propeller because of their inaccessibility from inside of the ship.

The theory of the reciprocity principle, which is known since the publication of Lord Rayleigh, ${ }^{2)}$ gives us the solution for this problem.

The vibro-acoustic reciprocity method, which was developed by Steenhoek, ten Wolde and followers, ${ }^{3-7)}$ makes it possible to determine the sound transfer functions from the ship structure into the surrounding water by measuring structural response to incident sound from the source.

The aim of this paper is to evaluate the validity of the vibro-acoustic reciprocity method for measuring the mechano-acoustical transfer function of ship structures.

2. Vibro-acoustic reciprocity principle

By the reciprocity principle, the transfer function from the force $F$ exerted on the surface of the structure to the sound radiated into water, as shown in Fig. 1, can be replaced by the reciprocal system shown in Fig. 2. The reciprocal relation of these two systems can be given by ${ }^{5)}$

$$
(\not p / F)_{U=0}=(v / U)_{F=0},
$$

where $F$ is the force exerted on the structure, $p$ is the radiated sound pressure, $v$ is the normal velocity of the plate excited by incident sound pressure from the source and $U$ is the volume velocity of the sound source, which is defined as the diaphragm velocity of the hydrophone times its effective diaphragm area. ${ }^{1)}$

Supposing that the velocity can be expressed by the sinusoidal form as $v=C \cdot \exp (-j \omega t)$, Eq. (2) can be written as

$$
(\not / F)_{U=0}=-(\alpha / j \omega U)_{F=0},
$$

where $\alpha$ is the acceleration of the surface vibration of the plate and $\omega$ is its angular frequency.

For the monopole source, the volume velocity can be approximated by ${ }^{8)}$

$$
j \omega U=\left(4 \pi r^{\prime} / \rho_{0}\right) p^{\prime},
$$

where $\rho_{0}$ is the mass density of water and $p^{\prime}$ is the sound pressure at a distance $r^{\prime}$ from the source.

Combining Eqs. (1), (3) and (4), we have

$$
H=\left(\rho_{0} / 4 \pi r^{\prime}\right),\left(\alpha / p^{\prime}\right) \text {. }
$$

By this equation, the mechano-acoustical transfer function can be determined by measuring the underwater sound source and the resultant acceleration on the ship structure.

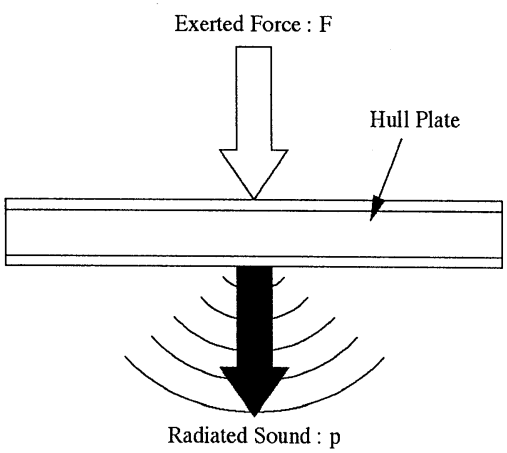

Fig. 1 Vibro-acoustic system for sound radiation.

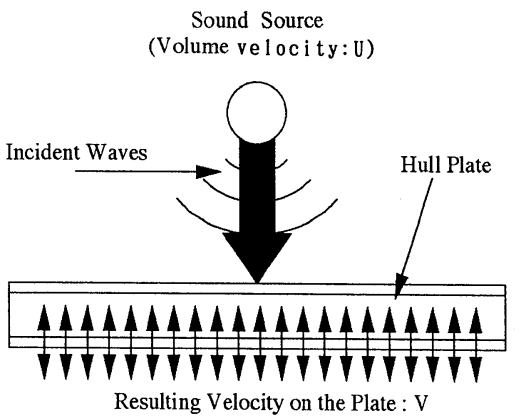

Fig. 2 Reciprocal system for sound radiation. 
3. Measurement of the mechano-acoustical transfer function for the steel vessel

To evaluate the validity of the vibro-acoustic reciprocity method for ship structures, the experiment was conducted in the anechoic water tank for the small vessel made of steel plate, as shown in Fig. 3.

The steel vessel used for the experiment has the following dimension: $1.5 \mathrm{~m}$ in width, $2.0 \mathrm{~m}$ in length and $1.0 \mathrm{~m}$ in height. The plate thickness is $5 \mathrm{~mm}$. Under the steel vessel, the sound source $S 0$ was set at the center of it with the separation of $0.5 \mathrm{~m}$ from its bottom and two hydrophones $\mathrm{H} 1$ and $\mathrm{H} 2$ were set on both sides of the sound source with the separation of $1.0 \mathrm{~m}$, as shown in Fig. 4.

To verify the reciprocity method, the direct measurement was also conducted by using the vibration exciter $F 0$ attached at the center of the floor in the steel vessel. Two accelerometers A1 and A2 were set on both sides

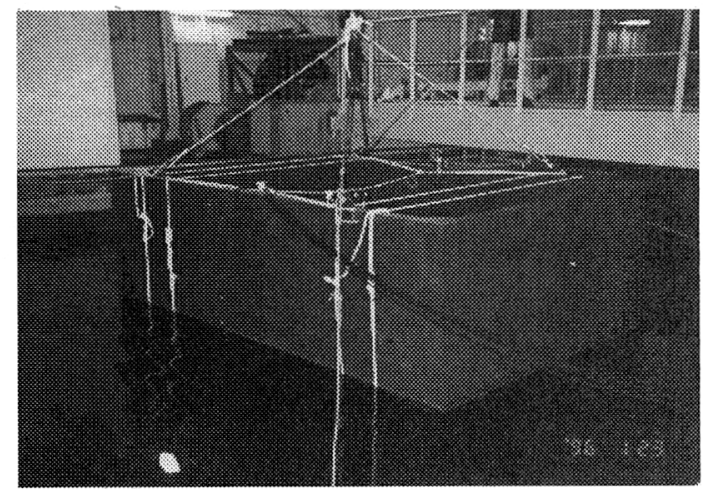

Fig. 3 Steel vessel used for the experiment. of it. Signals from these sensors were processed by the FFT analyzer. Figure 5 shows the sound pressure levels from the source measured by the hydrophone $\mathrm{H} 1$ and the resultant acceleration levels measured by the accelerometer $\mathrm{Al}$ on the plate in the reciprocal experiment.

The mechano-acoustical transfer function for the steel plate is calculated from the following equations, de-
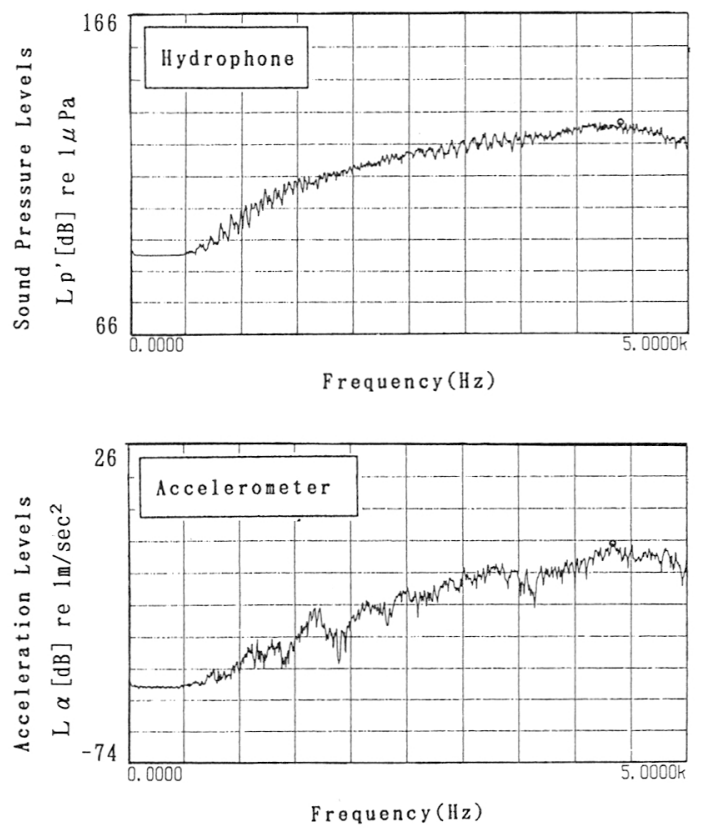

Fig. 5 Power spectra obtained by the reciprocal experiment.

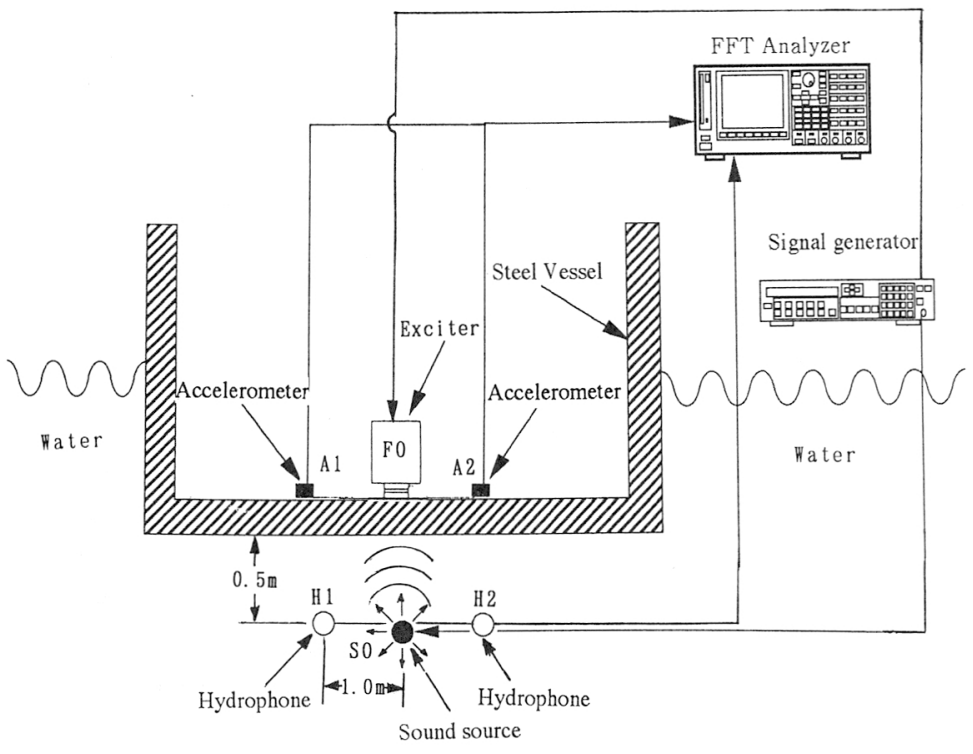

Fig. 4 Schematic diagram of the experimental system. 


\section{T. MUSHA and T. KIKUCHI : VALIDITY OF THE VIBRO-ACOUSTIC RECIPROCITY METHOD}

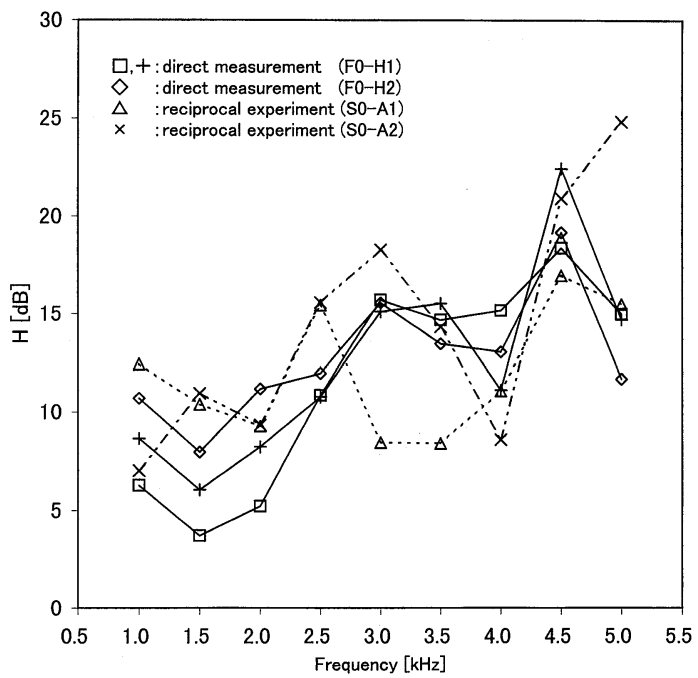

Fig. 6 Measured values of the mechanoacoustical transfer function by the direct measurement and the reciprocal experiment.

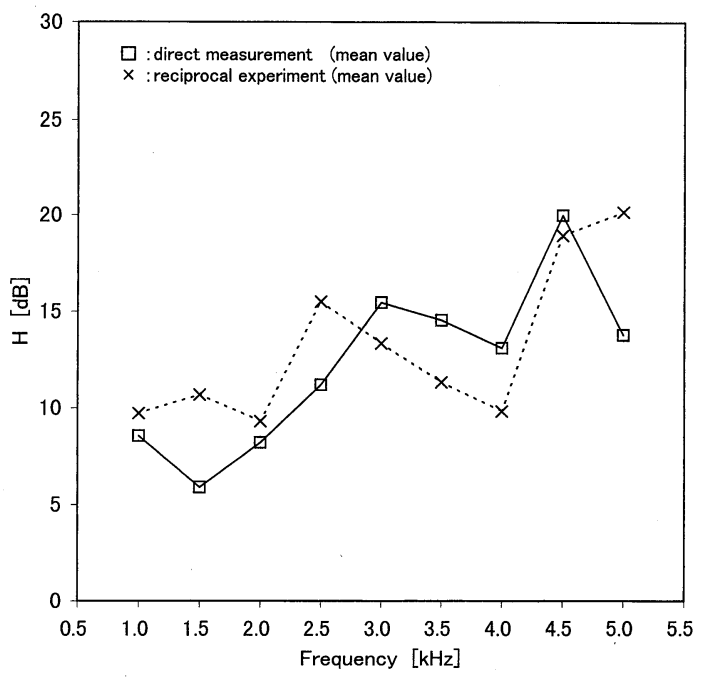

Fig. 7 Mean values of the mechano-acoustical transfer function of the steel plate by the direct measurement and the reciprocal experiment.

rived from Eqs. (1) and (5), for the direct measurement and the reciprocal experiment respectively :

(Direct measurement)

$$
H=L_{P}-L_{F}-120,
$$

where $H$ is the transfer function in $\mathrm{dB}, L_{\mathrm{p}}$ is the radiated sound pressure level in $\mathrm{dB}$ re $1 \mu \mathrm{Pa}$ and $L_{\mathrm{F}}$ is the applied force level in $\mathrm{dB}$ re $1 \mathrm{~N}$.

(Reciprocal experiment)

$$
H=L_{\alpha}-L_{p^{\prime}}+158,
$$

where $L_{a}$ is the resultant acceleration level in $\mathrm{dB}$ re 1 $\mathrm{m} / \mathrm{s}^{2}$ and $L_{p^{\prime}}$, is the sound source level in $\mathrm{dB}$ re $1 \mu \mathrm{Pa}$.

Figure 6 shows the transfer function data obtained by the direct measurement and the reciprocal experiment. In Fig. 7, the mean value of them are also presented.

From these results, it is seen that the mechanoacoustical transfer function by the reciprocity method almost agrees with the measurement data obtained by the direct excitation on the plate.

\section{Conclusion}

From this study, it is seen that the vibro-acoustic reciprocity method is applicable for measuring the mechano-acoustical transfer function for ship structures.

\section{Acknowledgement}

The authors wish to thank Mr.Mutuo Takashima and his staff of JRC Tokki Co., Ltd, for co-operating to conduct the experiment.

\section{References}

1) J. G. van Bakel and H. F. Steenhoek, "On recent ship noise transmission experiments using the reciprocity method," Tech. Univ. Delft Publ. TNO Institute of Applied Physics, 297-303 (1982).

2) Lord Rayleigh, "On the application of the principle of reciprocity to acoustics," Proc. R. Soc. 25, 118-122 (1876).

3) H. F. Steenhoek and T. ten Wolde, "The reciprocam measurement of mechanical-acoustical transfer functions," Acustica 23, 301-305 (1970).

4) T. ten Wolde, "Reciprocity experiments on the transmission of sound in ships," Ph. D. Thesis, Tech.Univ. Delft Publ. Hooglond \& Waltman, Delft (1973).

5) T. ten Wolde, "On the validity and application of reciprocity in acoustical, mechano-acoustical and other systems," Acustica 28, 23-32 (1973).

6) T. ten Wolde, J. W. Verheij, and H. F. Steenhoek, "Reciprocity method for the measurement of mechano-acoustical transfer functions," J. Sound Vib. 42, 49-55 (1975).

7) T. Musha, I., Abe, A. Shinohara, and M. Takashima, "Evaluation of acoustic radiation efficiency for hull plate using the reciprocity method," JSME Int. J. Ser. C 37, 612-615 (1994).

8) R. D. Ford, Introduction to Acoustics (Elsevier Publ. Co. Ltd., London, 1970). 